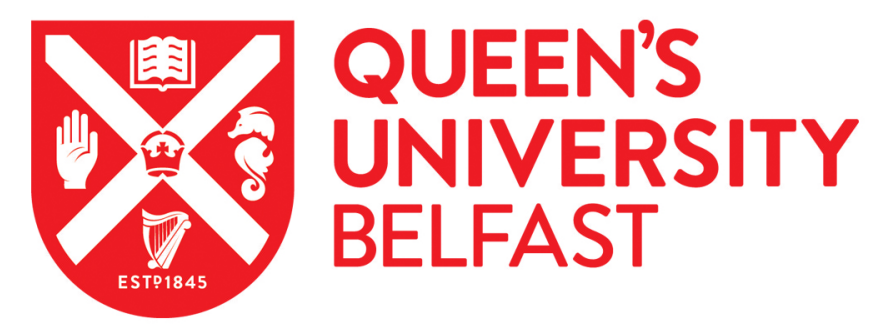

\title{
Production of ultracold hydrogen and deuterium via Doppler-cooled Feshbach molecules
}

Lane, I. C. (2015). Production of ultracold hydrogen and deuterium via Doppler-cooled Feshbach molecules. Physical Review A (Atomic, Molecular, and Optical Physics), 92(02), [022511].

https://doi.org/10.1103/PhysRevA.92.022511

Published in:

Physical Review A (Atomic, Molecular, and Optical Physics)

Document Version:

Publisher's PDF, also known as Version of record

Queen's University Belfast - Research Portal:

Link to publication record in Queen's University Belfast Research Portal

Publisher rights

@2015 American Physical Society

\section{General rights}

Copyright for the publications made accessible via the Queen's University Belfast Research Portal is retained by the author(s) and / or other copyright owners and it is a condition of accessing these publications that users recognise and abide by the legal requirements associated with these rights.

Take down policy

The Research Portal is Queen's institutional repository that provides access to Queen's research output. Every effort has been made to ensure that content in the Research Portal does not infringe any person's rights, or applicable UK laws. If you discover content in the Research Portal that you believe breaches copyright or violates any law, please contact openaccess@qub.ac.uk. 


\title{
Production of ultracold hydrogen and deuterium via Doppler-cooled Feshbach molecules
}

\author{
Ian C. Lane* \\ School of Chemistry and Chemical Engineering, Queen's University Belfast, Stranmillis Road, Belfast BT95AG, United Kingdom
}

(Received 19 February 2015; revised manuscript received 11 June 2015; published 24 August 2015)

\begin{abstract}
A counterintuitive scheme to produce ultracold hydrogen via fragmentation of laser cooled diatomic hydrides is presented where the final atomic $\mathrm{H}$ temperature is inversely proportional to the mass of the molecular parent. In addition, the critical density for formation of a Bose-Einstein condensate (BEC) at a fixed temperature is reduced by a factor $\left(m_{H} / m_{M H}\right)^{3 / 2}$ over directly cooled hydrogen atoms. The narrow Feshbach resonances between a ${ }^{1} S_{0}$ atom and hydrogen are well suited to a tiny center of mass energy release necessary during fragmentation. With the support of $a b$ initio quantum chemistry, it is demonstrated that $\mathrm{BaH}$ is an ideal diatomic precursor that can be laser cooled to a Doppler temperature of $\sim 26 \mu \mathrm{K}$ with just two rovibronic transitions, the simplest molecular cooling scheme identified to date. Preparation of a hydrogen atom gas below the critical BEC temperature $T_{c}$ is feasible with present cooling technology, with optical pulse control of the condensation process.
\end{abstract}

DOI: 10.1103/PhysRevA.92.022511 PACS number(s): 33.70.Ca, 37.10.Mn, 34.20.Cf, 31.15.vn

\section{INTRODUCTION}

Hydrogen is the most abundant element in nature and arguably the single most important atomic system for testing our understanding of the physical universe. Naturally the fundamental need for accurate measurements on the properties of hydrogen has meant great effort has been expended in bringing the techniques of laser cooling [1] and trapping to this unique system, but, with the lowest energy transition in the vacuum UV (VUV), the recoil temperature $T_{r}$ is ultimately high [2] and the required laser technology is currently unavailable. In 1998, a Bose-Einstein condensate (BEC) of hydrogen was achieved in a cryogenic (He) environment [3], but the final atom density was very high and the optical access was limited [4]. Furthermore, this technique has thus far not produced a degenerate fermionic gas of deuterium. In this paper a method to produce ultracold $\mathrm{H}$ or $\mathrm{D}$ atoms via the fragmentation of laser-cooled hydrides and deuterides is outlined. In particular, the benefits of a counterintuitive scheme, whereby the lowest possible $\mathrm{H}$ or $\mathrm{D}$ temperatures are produced by pairing with a significantly heavier element, are discussed.

Despite significant progress in both technique and technology, large parts of the periodic table remain closed to experiments in ultracold science. Previously a method has been proposed to produce ultracold samples of carbon [5] and fluorine [6], two elements that are currently difficult to cool to sub-mK temperatures, by the photodissociation of laser cooled diatomics ( $\mathrm{CH}$ and $\mathrm{BeF}$, respectively). This will be summarized here in Sec. II A. The present application of this technique (Fig. 1) significantly extends the method by also exploiting an additional kinematic effect, for the final velocity distributions of the atomic fragments are described by lower temperatures than the initial molecular gas (Sec. II B). The essential criteria required in the molecular parent for it to work are discussed in Sec, II C and the Group II hydride $\mathrm{BaH}$ appears to be a strong candidate. To investigate its suitability further, $a b$ initio calculations are performed (Sec. III A) on $\mathrm{BaH}$ to determine the electronic potentials, particularly at long

*i.lane@qub.ac.uk range, as well as the transition dipole moments (Sec. III B). This allows the viability of possible laser cooling pathways to be investigated (Sec. III C) and finally the possibility of optical transfer from a ground to an excited vibronic level close to the dissociation limit (Sec. III D). Finally, the reduced momentum of the hydrogen product increases dramatically the de Broglie wavelengths of these atoms. In Sec. IV it is proposed that the boost in effective phase space occupied by each atom can dramatically lower the critical density required for the formation of a Bose-Einstein condensate (BEC), suggesting that the creation of a quantum gas of hydrogen atoms can be optically controlled.

\section{SLOW ATOMS VIA PHOTOFRAGMENTATION}

\section{A. Zero-energy fragmentation of diatomics}

This paper concentrates on the fragmentation of a diatomic into component atoms. The final laboratory frame velocity $V_{A}$ of a fragment atom $\mathrm{A}$ is the vector sum of the center of mass velocity $v_{A}$ of atom and the laboratory frame velocity $V_{A B}$ of the parent molecule (this is the velocity of the center of mass in the laboratory frame). If the center of mass velocities $v_{\text {c.m. }}$ of the fragments are significantly smaller than the initial velocity $V_{A B}$ of the parent molecule, the laboratory frame fragment velocity becomes $V_{A}=V_{A B}+v_{A} \approx V_{A B}$. In a typical photodissociation experiment, a short optical pulse is used to excite a parent molecule to an excited electronic state. Before this state can decay radiatively back to the ground state intact, it undergoes fragmentation, either directly along a repulsive potential or via coupling to a repulsive state through the process of predissociation. As these states are at elevated energies, the resulting photofragment $\mathrm{A}$ is produced with large $v_{A}$, the laws of kinematics determining the partitioning of kinetic energy between it and its partner. In this case, the magnitude of $v_{A}$ is too great to ensure $V_{A} \approx V_{A B}$.

Minimization of the energy released in the fragmentation step with respect to the initial (thermal) kinetic energy of the molecules requires the fragmentation to take place at the dissociation limit of an electronic state. If a rovibronic level lies very close to the dissociation limit, an external electric or magnetic field can be applied to sweep the occupied 


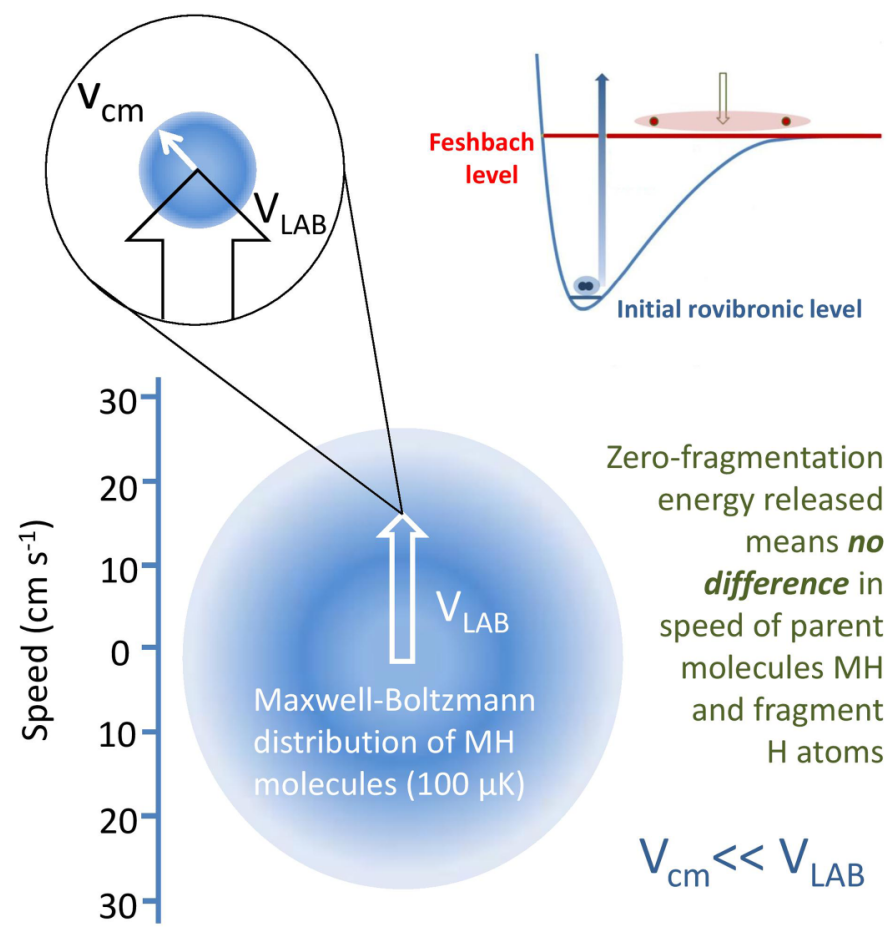

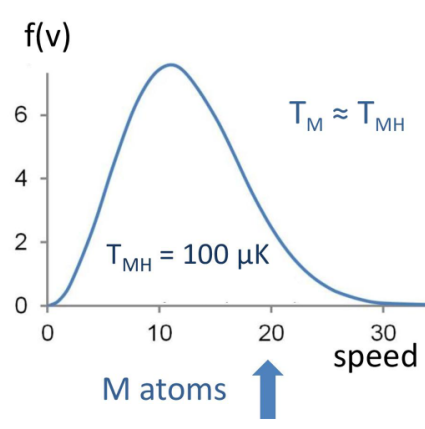

Fragments have same velocity distribution

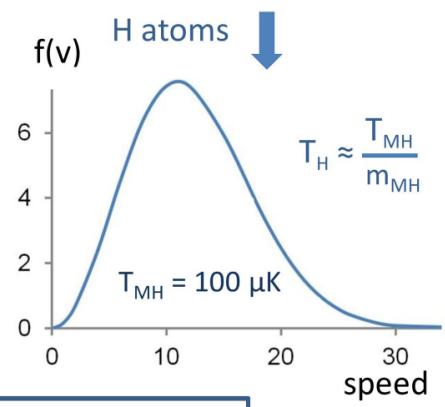

Hence $\mathrm{H}$ atom fragments colder than the parent $\mathrm{MH}$

FIG. 1. (Color online) The dynamics of zero-energy fragmentation in a molecular gas: For gaseous molecules at temperature $T$ moving with the average velocity $V_{\text {rms }}$, the center-of-mass velocity of the fragmentation products is assumed to be considerably smaller than the lab frame velocities of the parents $V_{\text {lab }}=V_{\text {rms }}$ (left panel). This is achieved through excitation via a Feshbach level (upper middle panel). The atomic fragments are consequently moving in the laboratory frame with the same velocity as the parent molecule. The gas of fragmentation products will therefore also have a Maxwell Boltzmann distribution of fragment speeds. However, the laboratory temperature that describes the fragment velocity distribution depends on the ratio of their mass to the mass of the parent (right panel). An extreme example is the production of $\mathrm{H}$ fragments from a hydride, such as $\mathrm{BaH}$. A very cold gas (here $T=100 \mu \mathrm{K}$ ) will obey the same dynamics, though the low energy fragmentation condition is more demanding to fulfill. All speeds in $\mathrm{cm} \mathrm{s}^{-1}$.

level across the dissociation limit and affect fragmentation into atoms. However, the typically poor Franck-Condon (FC) factors to populate such a level from the ground rovibrational state and the short lifetime of the excited electronic states can make this difficult, but there are examples of such a similar process in the form of ZEKE (zero electron kinetic energy) [7] spectroscopy, which excites Rydberg states close to the ionization potential of a molecule before applying an external electric field to ionize them, producing photoelectron spectra of very high resolution. In the case of photofragmentation, the problem of a short lifetime can be addressed by choosing the lowest dissociation limit, and the restrictions of both selection rules and FC factors can be circumvented by using two or more optical pulses to excite the dissociating level via an excited electronic state. If the pulse energies are strong enough, transfer of population within the ground state potential [8] alone may even be possible via vibrational overtones. It is well understood in the field of reaction dynamics that the experimental control possible in a "half-collision" such as photodissociation far exceeds that possible investigating a binary (full) collision.

The dissociated atoms have zero-kinetic energy in the center of mass frame only. To ensure this is also the case in the laboratory frame the molecular parents themselves must be moving very slowly, requiring gas temperatures into the
sub-Kelvin. The most effective method to fulfill this condition is direct Doppler cooling with lasers [9], but this requires a diagonal electronic transition and an excited state that does not suffer significant losses, either via radiative decay to alternate electronic states or by predissociation [5]. Stark [10] and Zeeman [11] deceleration of molecules can achieve temperatures into the $\mathrm{mK}$ range but not into the ultracold $(<1 \mathrm{mK})$. While it is advantageous to use a parent molecule that can be Doppler cooled in order to ensure as low an initial gas temperature as possible, the present application to hydrogen atoms is not necessarily limited to just these molecules.

The initial step in achieving low-kinetic energy fragmentation is to transfer the molecular population coherently from the final vibronic state in the cooling cycle into a Feshbach [12] resonance, the reverse of the often used magnetoassociation technique [13] for producing ground-state cold molecules from laser-cooled atoms. The fragile molecular bond is then destroyed by altering an external field. For this paper this field will be assumed to be magnetic for this is the primary tool [14] used by experimental groups. The important thing to note is that the energy scale associated with the magnetic Feshbach resonances [15] is orders of magnitude smaller than those found in ordinary thermally driven or photochemical bond breaking as such processes involve energy releases equivalent to tens or even hundreds of $\mathrm{kJ} \mathrm{mol}^{-1}$. By 
contrast, the relevant energy scale in the ultracold region is $1 \mu \mathrm{K} \approx 23 \mathrm{kHz} \approx 8.34 \mu \mathrm{J} \mathrm{mol}^{-1}$. Typically, Feshbach resonance widths in a magnetic field are defined by the gradient $\frac{d E}{d B} \approx \delta \mu B$, where $\delta \mu$ is the difference in molecular and atomic dipole moments, while a Bohr magneton corresponds to $\mu_{B}=1.4 \mathrm{kHz} / \mathrm{mG}\left(1 \mathrm{G}=10^{-4} \mathrm{~T}\right)$.

\section{B. Cooling via fragmentation}

Now to the case of a heavy metal atom $\mathrm{M}$ bonded to a much lighter atom $\mathrm{A}$, the latter difficult to laser cool by conventional methods. The laws of mechanics dictate that when the fragmentation takes place the lighter fragment carries the greatest share of the available kinetic energy. Therefore, the heaviest fragment receives the smallest kinetic boost from the fragmentation step and is the most likely candidate for a negligible change in velocity $V_{M} \approx V_{M A}$, particularly from a laser cooled parent (very small $V_{M A}$ ). Clearly the smaller the velocity boost, the closer the final velocities of both fragments to that of the parent molecules and in the limit of a very small energy release even $V_{A} \approx V_{M A}$. If the lighter atom is hydrogen, when $v_{H} \ll V_{M H}$ it is possible to exploit the large disparity in atomic masses to radically modify the final momentum of the cold hydrogen fragment, for although the final laboratory frame velocities are $V_{H} \approx V_{M} \approx V_{M H}$, their momenta and associated de Broglie wavelengths are significantly different:

$$
P_{H}=\frac{m_{H}}{m_{M H}} P_{M H} \approx \frac{1}{m_{M H}} P_{M H} .
$$

Consequently, the more massive the nonhydrogen atom (and hence the diatomic), the greater is the subsequent increase in the de Broglie wavelength of the hydrogen atoms.

An alternative interpretation is the creation of two different atomic gases after fragmentation, initially with two distinct temperatures $T_{H}$ and $T_{M}$. Of course all the molecules in any thermal sample will not share the same velocity but a range of speeds given by the Maxwell-Boltzmann distribution (for particles of mass $m$ )

$$
f(V) d V=\left(\frac{m}{2 \pi k T}\right)^{3 / 2} 4 \pi V^{2} e^{\frac{-m V^{2}}{2 k T}} d V .
$$

When the molecule fragments, as long as $v_{H} \ll V_{M H}$, the fragments formed will have the same velocity distribution as the parent molecules. However, they will have very different momenta and although the distribution of fragment velocities will be little changed, it will correspond to a lower temperature than the parent molecules due to the ratio $m / T$ in the preexponential and exponential terms. The hydrogen atom, being lighter, effectively has a significantly lower temperature than both the parent molecules and the hydride partner. The total thermal energy does not change as there are now twice as many particles present in the same volume:

$$
\begin{aligned}
3 k T & =m_{M H} V^{2} \stackrel{\text { fragment }}{\longrightarrow} m_{H} V^{2}+m_{M} V^{2} \\
& =\left(m_{H}+m_{M}\right) V^{2}=m_{M H} V^{2}=3 k T .
\end{aligned}
$$

The heavier the partner, the greater the disparity between the $\mathrm{H}$ atom temperature formed and that of the parent, while the partner element will approach the initial temperature of the molecular gas. Thus, effectively, cooling of the hydrogen component of the molecules has been achieved through fragmentation $\left(T_{H} \ll T_{M} \approx T_{M H}\right)$.

The width of the magnetic Feshbach resonance [16] $\Delta(B)$ is a manifestation of the Feshbach linewidth $\Gamma(E)$,

$$
\Delta(B)=\frac{\Gamma(E)}{2 k a_{\mathrm{bg}} \delta \mu} .
$$

$a_{\mathrm{bg}}$ is the background scattering length determined by the potential of the dissociating state (the ground state), while $k$ is the wave vector describing the colliding bodies. Note, however, that the magnitude of $\Gamma(E)$ itself may also be dependent on the magnetic field strength. For a dissociating molecule, the wave vector is determined by the momentum of the fragments in the center of mass frame

$$
\Delta(B)=\frac{\Gamma(E) h}{4 \pi\left|m_{H} v_{H}\right| a_{\mathrm{bg}} \delta \mu}=\frac{\Gamma(E) h}{4 \pi\left|m_{M} v_{M}\right| a_{\mathrm{bg}} \delta \mu} .
$$

Identifying the linewidth $\Gamma(E)$ as the width of the center of mass energy release in the threshold dissociation step and rearranging

$$
\Delta(B)=\frac{\Gamma(E)^{1 / 2} h}{4 \pi\left(2 m_{\mu}\right)^{1 / 2} a_{\mathrm{bg}} \delta \mu} \approx \frac{\Gamma(E)^{1 / 2} h}{4 \pi\left(2 m_{H}\right)^{1 / 2} a_{\mathrm{bg}} \delta \mu} .
$$

This result follows because for any diatomic hydride beyond the second row the reduced mass $m_{\mu} \approx m_{H}$.

It is well known that the reaction enthalpy can distort [17] the Maxwellian velocity distribution, but this is minimized if miniscule fragmentation energies are involved. This requirement to disturb the laboratory frame velocity of atom A as little as possible sets an upper limit to the linewidth $\Gamma(E)$ in the fragmentation of a diatomic MA with thermal (kinetic) energy $E_{M A}$,

$$
\Gamma(E) \leqslant \frac{m_{A}}{m_{M}} E_{M A} .
$$

This condition is particularly difficult to fulfill for the lighter atom and naturally most severe for hydrogen. However, for Feshbach widths of $<100 \mathrm{mG}$ this condition can still be satisfied for hydrogen products from a molecular gas at sub-mK temperatures. Feshbach resonances of width $10 \mathrm{mG}$ or narrower [16] are not uncommon, ensuring the energy released in the fragmentation step is on a sub- $\mu \mathrm{K}$ energy scale. A similar kinematic effect can be achieved for other light atoms and naturally for the heavier isotopes of hydrogen (deuterium and tritium). The final "temperatures" possible will not be as low as that possible for hydrogen, but equally the energy condition above will not be as severe.

The nascent hydrogen velocity distribution will only be approximately Maxwellian and the extent of the deviation depends on the magnitude of $\Gamma(E)$. Once the ultracold hydrogen gas has been formed, it will begin to thermalize through collisions within the trap, not only between pairs of hydrogen atoms but with the equivelocity partner atoms (laboratory frame) also produced in the fragmentation. The opportunity to manipulate this thermalization process, for example by selectively removing the heavier atoms via external fields, is a further unique feature of this photofragmentation method. 


\section{Molecular candidates}

Hydride molecules suitable for the formation of cold hydrogen must satisfy four basic requirements, namely (1) they can be cooled, preferably by laser cooling into the ultracold; (2) they possess very narrow Feshbach resonances to minimize the energy release in the photoinduced fragmentation step; (3) they have a large molecular mass to maximize the kinematic cooling effect; and finally (4) there exists an efficient pathway between the initial quantum state of the cooled molecules and the final Feshbach resonance.

The fourth and final conditions require an electronic energy structure that facilitates the efficient transfer of the molecular population from a deeply bound quantum state (the final rovibrational state following laser cooling) into a loosely bound Feshbach state prior to dissociation. Stimulated rapid adiabatic passage (STIRAP) [18], a fully coherent optical procedure for population transfer, has been used previously for the formation of ultracold molecules [14] from cold atoms. An excited molecular electronic state is required to act as a bridge between the initial and target quantum states, a difficult condition to fulfill sometimes as evidenced by recent work on producing ultracold fluorine atoms from BeF [6], where large transition energies and low lying Rydberg states can complicate the available pathways.

The heavier hydrides of alkaline earth metals are potential candidates that fulfill all four requirements and therefore open the door to the production of an ultracold gas of hydrogen or its isotope deuterium, the latter of which has yet to be cooled to degeneracy. Recently, Gao and co-workers [19] surveyed the laser cooling prospects of the alkaline earth hydrides (except $\mathrm{RaH})$ but concentrated efforts on the $A^{2} \Pi \leftarrow X^{2} \Sigma^{+}$Franck Condon (FC) factors only despite other factors playing a key role. The lightest examples, $\mathrm{BeH}$ and $\mathrm{MgH}$, for example, can be ignored as they are both prone to predissociation [5] on both the $A^{2} \Pi \leftarrow X^{2} \Sigma^{+}$and $B^{2} \Sigma^{+} \leftarrow X^{2} \Sigma^{+}$cooling transitions (Fig. 2), which introduces another source of
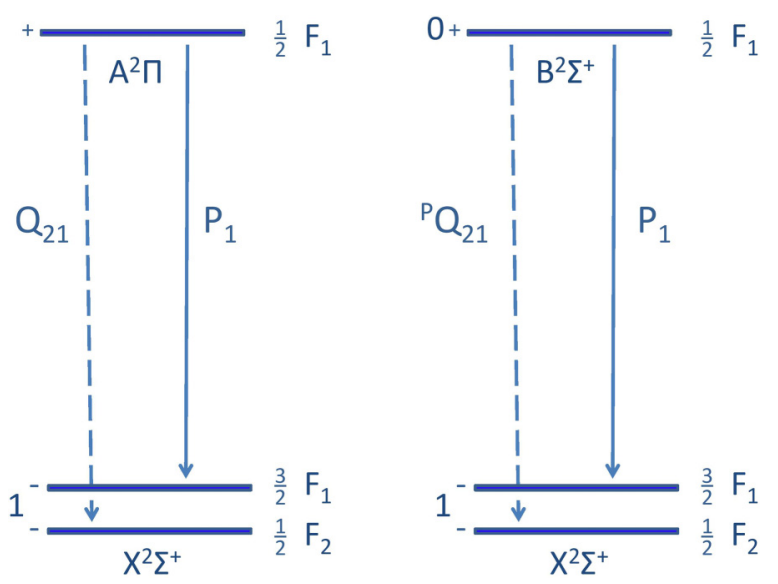

FIG. 2. (Color online) The rotational lines involved in laser cooling molecules on the $A^{2} \Pi-X^{2} \Sigma^{+}$and $B^{2} \Sigma^{+}-X^{2} \Sigma^{+}$vibronic transitions. Note the Hund's case $(a)$ labeling appropriate for a low rotational state and the basic similarity between the two transitions as both require an excited rotational state with $J=1 / 2$. The splitting present in the lower $N=1$ level is due to spin-rotation coupling and is usually on the order of a few $\mathrm{GHz}$ for heavy hydrides. molecular loss. The situation is rather less clear for $\mathrm{CaH}$, but recent $a b$ initio [20] and experimental work strongly suggests that only the lowest vibrational level of the $A^{2} \Pi$ state does not undergo predissociation, and so $\mathrm{CaH}$ will not be considered here either. While $\mathrm{SrH}$ does appear to be a suitable candidate, the superior mass of $\mathrm{BaH}$ means this diatomic is the basis of the present study. In previous work this group has shown [5,21] that ab initio quantum chemistry can be used to identify possible cooling schemes for cooling diatomics. In the present work there is a wealth of experimental data too and this information is combined with high level ab initio calculations to develop an accurate a set of potential curves for $\mathrm{BaH}$ and to obtain the bound rovibronic levels, spectroscopic constants, FC factors, and radiative lifetimes.

\section{AB INITIO CALCULATIONS}

\section{A. Computational methods}

The cc-pV5Z (V5Z) basis set is used for $\mathrm{H}$ and all calculations were performed using the MOLPRO suite [22] of programs. Two barium basis sets were tested in this study: the first a ten electron type and the second a two electron one. Both used an ECP to simulate the inner electrons. However, in the case of the two electron set an $l$-independent core polarization potential (CPP) was also included. The principle criterion used for the choice of the basis set was the ability to correctly model the asymptotic energies of the molecular potentials (corresponding to the lowest electronic states of the $\mathrm{Ba}$ atom). Despite slashing the computational time, the second set proved superior in calculating the asymptotic energies (the CPP component was crucial in this) and therefore only these results are presented. The barium basis set used is very compact and therefore should be suitable for calculations of the relevant collisional potentials involving the hydride as well, such as $\mathrm{BaH}$ with $\mathrm{Ba}$ atoms or bimolecular collisions involving the dimers. The details of the basis set and CPP are shown in Fig. 3.

The electronic states are found using complete active space SCF (CASSCF) wave functions [23] based on the valence

\author{
BARIUM $\quad(7 \mathrm{~s}, 6 \mathrm{p}, 6 \mathrm{~d}, 2 \mathrm{f})$ \\ s, BA , 0.791740, $0.316178,0.066565,0.026990,0.013495$, \\ $0.00907737,0.003$ \\ p, BA , 0.685598, 0.338376, 0.073598, 0.034232, 0.015836 , \\ 0.003 \\ d, BA , 1.377363, 0.785942, 0.492827, 0.168078, 0.058334, \\ 0.02 \\ f, BA, $0.40,0.1$ \\ ECP,Ba,54,3,0; \\ 1; 2,1.000000,0.000000; \\ 1; 2,0.6356,16.710; \\ $1 ; 2,0.3214,4.874$ \\ 1; 2,0.2403,-0.8427; \\ cpp,init,1; \\ ba,1,10.17,,0.243;
}

FIG. 3. Details of the compact barium atom basis set used. 


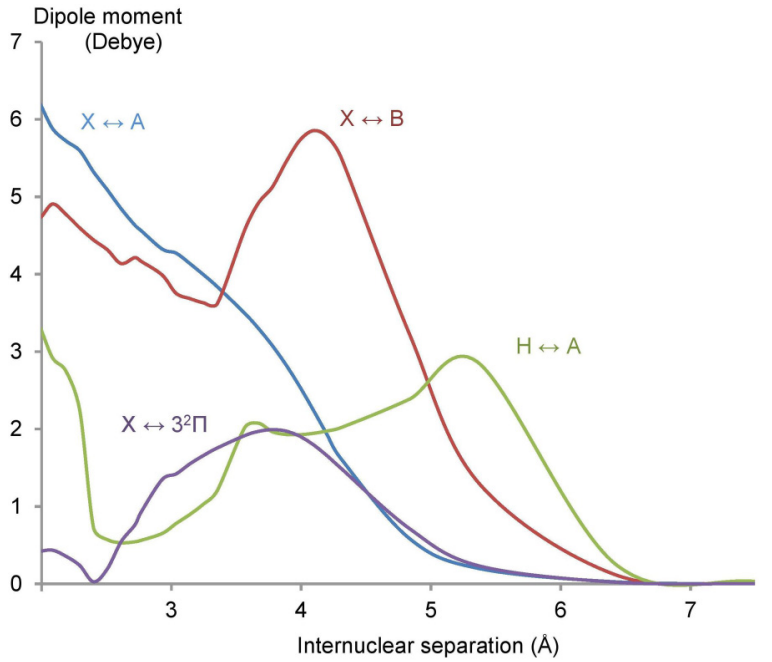

FIG. 4. (Color online) Transition dipoles as function of distance (CASSCF).

orbital space $5 a_{1}, 2 b_{1}, 2 b_{2}$, and $2 a_{2}$ (5222) with no core orbitals. Dynamic electron correlation was calculated [24] by multireference configuration interaction (MRCI), using the relaxed Davidson correction [25] throughout. These calculations were augmented with additional points using the larger active spaces 6532 (for the Rydberg component of the $\Pi$ states) and 7432 (for the $\Sigma^{+}$Rydberg states). Only states of doublet multiplicity were calculated. The $a b$ initio data points were fitted using the LEVEL program [26] (version 8.0) to obtain the bound rovibronic levels, spectroscopic constants, FC factors, and radiative lifetimes.

\section{B. Ab initio potentials}

The main aim of the calculations was to find the relevant transition dipoles (Fig. 4) between those electronic states that affect the rate of cooling, the presence of losses in the cooling cycle, and the efficiency of the STIRAP process that have not been determined in previous calculations (save [17] for $A^{2} \Pi-X^{2} \Sigma^{+}$). In addition, unlike previous work, the potentials outside the spectroscopically studied Franck-Condon region were computed as accurately as possible by using the correct long range expansion (based on the van der Waals coefficient $C_{6}$ ) in the fitting routine where possible. This is important for accurately finding a strong STIRAP pathway. The present calculations do not include relativistic effects but for completeness the correlations between molecular electronic states and the spin-orbit levels of the Ba atom are shown in Table I. The calculated atomic asymptotes (Table II) are in good agreement with experiment.

Ramanaish and Lakishman [27] have previously computed FC factors based on RKR potentials from spectroscopic work prior to 1982 . The FC factors for the $A^{2} \Pi-X^{2} \Sigma^{+}$transition in particular were strongly diagonal, with $f_{00}>0.99$. However, the RKR potential from Ramanaish and Lakishman for the $X^{2} \Sigma^{+}$state is not consistent with the more (2013) recent molecular constants from Ram and Bernath [28] (for example, using Ram's work the $v=10$ level lies at $10667 \mathrm{~cm}^{-1}$ and not $9733.60 \mathrm{~cm}^{-1}$ as suggested by Ramanaich). By contrast, the
TABLE I. The relevant molecular state, asymptotic limits, and most likely correlations for the lowest states of $\mathrm{BaH}$.

\begin{tabular}{lcc}
\hline \hline $\begin{array}{l}\text { Atomic } \\
\text { limit }\end{array}$ & $\begin{array}{c}\text { Experimental } \\
\text { asymptotic limit }\end{array}$ & $\begin{array}{c}\text { Molecular } \\
\text { states }\end{array}$ \\
\hline $\mathrm{Ba}\left({ }^{1} S_{0}\right)+\mathrm{H}\left({ }^{2} S_{g}\right)$ & 0 & ${ }^{2} \Sigma_{1 / 2}^{+}$ \\
$\mathrm{Ba}\left({ }^{3} D_{1}\right)+\mathrm{H}\left({ }^{2} S_{g}\right)$ & 9034 & ${ }^{2} \Sigma_{1 / 2}^{+},{ }^{2} \Pi_{1 / 2},{ }^{2} \Delta_{3 / 2}$ \\
$\mathrm{Ba}\left({ }^{3} D_{2}\right)+\mathrm{H}\left({ }^{2} S_{g}\right)$ & 9216 & ${ }^{2} \Pi_{3 / 2},{ }^{2} \Delta_{5 / 2}{ }^{4} \Sigma_{1 / 2}^{+},{ }^{4} \Pi_{1 / 2},{ }^{4} \Delta_{3 / 2}$ \\
$\mathrm{Ba}\left({ }^{3} D_{3}\right)+\mathrm{H}\left({ }^{2} S_{g}\right)$ & 9597 & ${ }^{4} \Pi_{1 / 2},{ }^{4} \Pi_{3 / 2}{ }^{4} \Pi_{5 / 2},{ }^{4} \Delta_{1 / 2}$, \\
$\mathrm{Ba}\left({ }^{1} D_{2}\right)+\mathrm{H}\left({ }^{2} S_{g}\right)$ & 11395 & ${ }^{2} \Pi_{3 / 2},{ }^{2} \Delta_{5 / 2},{ }^{2} \Sigma_{1 / 2}^{+},{ }^{2} \Pi_{1 / 2},{ }^{2} \Delta_{3 / 2}$ \\
$\mathrm{Ba}\left({ }^{3} P_{0}\right)+\mathrm{H}\left({ }^{2} S_{g}\right)$ & 12266 & ${ }^{2} \Pi_{1 / 2}$ \\
$\mathrm{Ba}\left({ }^{3} P_{1}\right)+\mathrm{H}\left({ }^{2} S_{g}\right)$ & 12637 & ${ }^{2} \Sigma_{1 / 2}^{+},{ }^{2} \Pi_{3 / 2},{ }^{4} \Pi_{1 / 2}$ \\
$\mathrm{Ba}\left({ }^{3} P_{2}\right)+\mathrm{H}\left({ }^{2} S_{g}\right)$ & 13515 & ${ }^{4} \Pi_{1 / 2},{ }^{4} \Pi_{3 / 2}{ }^{4} \Pi_{5 / 2},{ }^{4} \Sigma_{1 / 2}^{+},{ }^{4} \Sigma_{3 / 2}^{+}$ \\
\hline \hline
\end{tabular}

RKR potential for the $A^{2} \Pi$ state is still in good agreement with the later experimental work [29] by Barrow and co-workers.

A combination of experimental data and the present $a b$ initio calculations was used to recalculate the relevant FC factors. When computing the FC factors and the radiative lifetimes we incorporated as much experimental data [27-31] as possible to refine the potentials. All the calculated potentials were thus first shifted by $+0.072 \AA$ so the calculated ground equilibrium bond length matched experiment, a step that will not alter the FC factors as it is the relative energies of the potentials with internuclear separation that is important. In addition, the potentials were then scaled by 0.98 to improve the vibrational separations and the rotational constants (Fig. 5) that will alter the $\mathrm{FC}$ factors slightly compared to the unaltered $a b$ initio potentials. The ground $X^{2} \Sigma^{+}$state dispersion $C_{6}$ coefficient was computed as 147.5(4) using the polarizability data of Derevianko et al. [32], and the excited state values were estimated for the purposes of fitting the potentials (see Supplemental Material [33]). We compared FC factors from our purely $a b$ initio potentials with a combination that included the RKR from the $A$ state instead of our ab initio data. In agreement with Ramanaish and Lakishman, the ab initio diagonal FC factors are larger for the $A^{2} \Pi-X^{2} \Sigma^{+}$transition than $B{ }^{2} \Sigma^{+}-X^{2} \Sigma^{+}$, but the magnitude of the $f_{00}$ value is reduced to just under 0.97 . This result compares well with the recent relativistic calculation [19] (0.971) from Gao et al. The other relevant details of the electronic potentials are shown in Table III and compared to available experimental data.

TABLE II. Experimental asymptotes [47] versus nonrelativistic ab initio values (in $\mathrm{cm}^{-1}$ ).

\begin{tabular}{lccr}
\hline \hline States & Experimental value & Ab initio & Error \\
\hline $\mathrm{Ba}\left({ }^{1} S\right)+\mathrm{H}\left({ }^{2} S\right)$ & 0 & 0 & 0 \\
$\mathrm{Ba}\left({ }^{3} D\right)+\mathrm{H}\left({ }^{2} S\right)$ & 9372 & 9324 & 48 \\
$\mathrm{Ba}\left({ }^{1} D\right)+\mathrm{H}\left({ }^{2} S\right)$ & 11395 & 11508 & -113 \\
$\mathrm{Ba}\left({ }^{3} P\right)+\mathrm{H}\left({ }^{2} S\right)$ & 13098 & 13372 & -274 \\
$\mathrm{Ba}\left({ }^{1} P\right)+\mathrm{H}\left({ }^{2} S\right)$ & 18060 & 17761 & 299 \\
\hline \hline
\end{tabular}




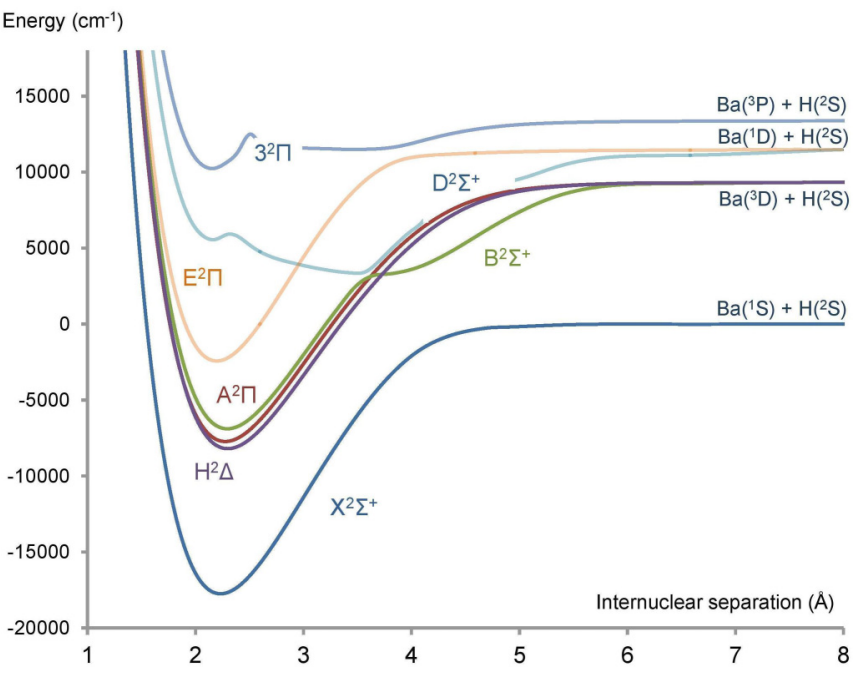

FIG. 5. (Color online) Calculated potentials for $\mathrm{BaH}$ and fitted using LEVEL 8.0.

Except for the lowest $J^{\prime}=1 / 2$ excited rotational state, the total Einstein $A$ coefficient for a transition $A_{v^{\prime} v^{\prime \prime}}\left(J^{\prime}\right)$ is given by the sum of $R$ and $P$ branch decays (or a strong $Q$ branch for the second $\Lambda$ doublet in the $A^{2} \Pi$ state), but for the most efficient rovibronic cooling transition only one decay path is present (Fig. 2). Thus, the lifetime of the $A^{2} \Pi v=0, J=1 / 2$ level is calculated as $148 \mathrm{~ns}$ with a corresponding Doppler temperature of $26 \mu \mathrm{K}$, consistent with the average $v=0 A^{2} \Pi$ lifetime [19] of $68.9 \mathrm{~ns}$ reported by Gao et al. It is worth noting that the recoil temperature for the $A^{2} \Pi v=0 \leftrightarrow X^{2} \Sigma^{+} v=0$ transition is just $125 \mathrm{nK}$, thanks to the long cooling wavelength and large molecular mass. The lifetime of the $\mathrm{BaH} B{ }^{2} \Sigma^{+} v=0$,

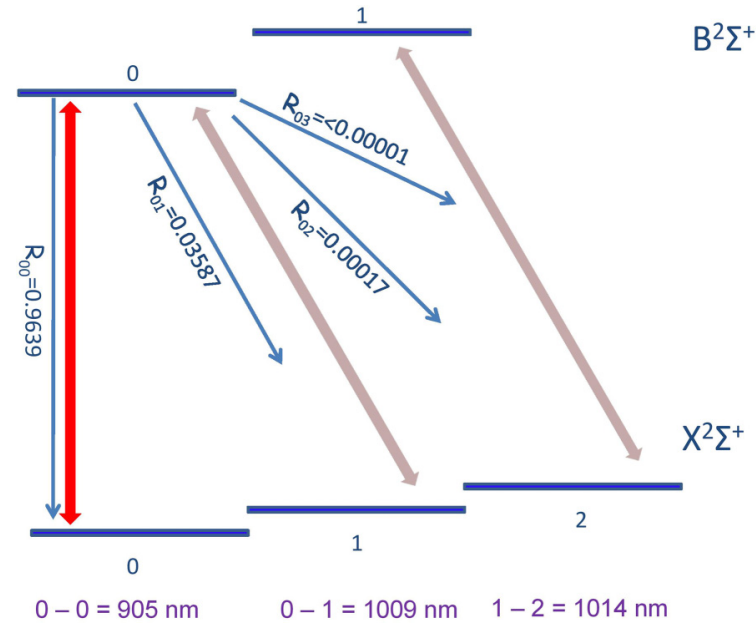

FIG. 6. (Color online) $\mathrm{BaH}$ cooling scheme based on the $B{ }^{2} \Sigma^{+}-X^{2} \Sigma^{+}$transition. The relative strengths of the photon decay pathways are labeled $R_{v^{\prime} v^{\prime \prime}}=A_{v^{\prime} v^{\prime \prime}} / \sum A_{v^{\prime} v^{\prime \prime}}$ rather than as FC factors. Cooling wavelengths indicated at the bottom.

$J=5^{1 / 2}$ level has been measured as $125 \pm 2 \mathrm{~ns}(31 \mu \mathrm{K})$ [34] and therefore the $B^{2} \Sigma^{+} v=0, J^{\prime}=1 / 2$ state will be on the order of twice that. The calculated FC factors for the $B^{2} \Sigma^{+} \leftrightarrow$ $X^{2} \Sigma^{+}$transition $f_{00}=0.933$ and $f_{01}=0.063$ are in excellent agreement with the experimental [34] values $f_{00}=0.94$ and $f_{01}=0.06$.

\section{Proposed cooling scheme}

With similar cooling rates available via both the $A^{2} \Pi$ and $B^{2} \Sigma^{+}$states, two cooling schemes (Figs. 6-8) are proposed, the crucial factor for successful cooling being

TABLE III. A comparison between the calculated constants (Ba basis defined earlier, $\mathrm{H}=\mathrm{V} 5 \mathrm{Z}$ basis set, this work) and experimental values (square bracketed values) for a variety of constants determined for the electronic states of the BaH radical. The $a b$ initio potentials were shifted and scaled as outlined in the text. $T_{e}$ is the energy of the potential minimum for the specified electronic state, $r_{e}$ is the internuclear separation at the minimum, $T_{v}$ is the energy of the vibrational level $v$ above the potential minimum (the calculated $T_{v}$ values assume $J=0$ ), and $B_{v}$ is the rotational constant for each vibrational level.

\begin{tabular}{|c|c|c|c|c|c|}
\hline $\mathrm{BaH}$ & $T_{e}\left(\mathrm{~cm}^{-1}\right)$ & $r_{e}(\AA)$ & $v$ & $T_{v}\left(\mathrm{~cm}^{-1}\right)$ & $B_{\mathrm{v}}\left(\mathrm{cm}^{-1}\right)$ \\
\hline \multirow[t]{4}{*}{$X^{2} \Sigma^{+}$} & 0 & 2.232 & 0 & $583.9[580.560]^{\mathrm{a}}$ & $3.354[3.3496]^{\mathrm{a}}$ \\
\hline & & {$[2.2319]^{\mathrm{a}}$} & 1 & $1731.6[1719.849]^{\mathrm{a}}$ & $3.297[3.2838]^{\mathrm{a}}$ \\
\hline & & & 2 & $2852.3[2830.166]^{\mathrm{a}}$ & $3.240[3.2179]^{\mathrm{a}}$ \\
\hline & & & 3 & $3945.8[3991.679]^{\mathrm{a}}$ & $3.183[3.1519]^{\mathrm{a}}$ \\
\hline$H^{2} \Delta$ & 9549.4 & 2.298 & 0 & $546.0[545.03]^{\mathrm{b}}$ & $3.161[3.1189]^{\mathrm{c}}$ \\
\hline$\left(A^{\prime 2} \Delta\right)$ & [9242.8] & [2.317] & 1 & $1616.9[1613.61]^{\mathrm{b}}$ & $3.101[3.0569]^{\mathrm{c}}$ \\
\hline \multirow[t]{2}{*}{$A^{2} \Pi$} & 10021.2 & 2.276 & 0 & $550.9[553.84]^{\mathrm{b}}$ & $3.223[3.2240]^{\mathrm{b}}$ \\
\hline & {$[9727.2]^{\mathrm{d}}$} & [2.249] & 1 & $1631.6[1636.73]^{\mathrm{b}}$ & $3.162[3.1526]^{\mathrm{b}}$ \\
\hline \multirow[t]{2}{*}{$B{ }^{2} \Sigma^{+}$} & 10858.0 & 2.295 & 0 & $547.6[540.58]^{\mathrm{d}}$ & $3.170[3.2335]^{\mathrm{d}}$ \\
\hline & {$\left[\begin{array}{lll}11 & 092.59\end{array}\right]^{\mathrm{d}}$} & [2.321] & 1 & $1621.3[1598.62]^{\mathrm{d}}$ & $3.110[3.1628]^{\mathrm{d}}$ \\
\hline \multirow[t]{2}{*}{$D^{2} \Sigma^{+}$} & 21091.7 & 3.504 & 0 & 315.64 & 1.507 \\
\hline & [21 885] & {$[3.22]$} & 1 & 825.64 & 1.692 \\
\hline \multirow[t]{2}{*}{$E^{2} \Pi$} & 15319.4 & 2.197 & 0 & $601.0[607.04]^{\mathrm{a}}$ & $3.462[3.4868]^{\mathrm{a}}$ \\
\hline & {$[14830.16]^{\mathrm{a}}$} & {$[2.1877]^{\mathrm{a}}$} & 1 & $1784.9[1797.62]^{\mathrm{a}}$ & $3.400[3.4145]^{\mathrm{a}}$ \\
\hline
\end{tabular}

a Reference [28].

${ }^{\mathrm{b}}$ Reference [29].

${ }^{\mathrm{c}}$ Reference [31].

${ }^{\mathrm{d}}$ Reference [30]. 
(a)

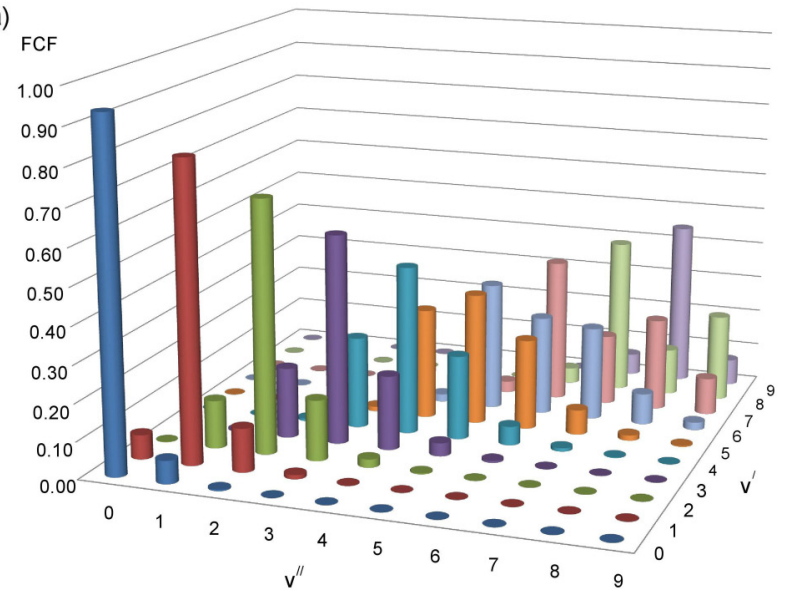

(b)

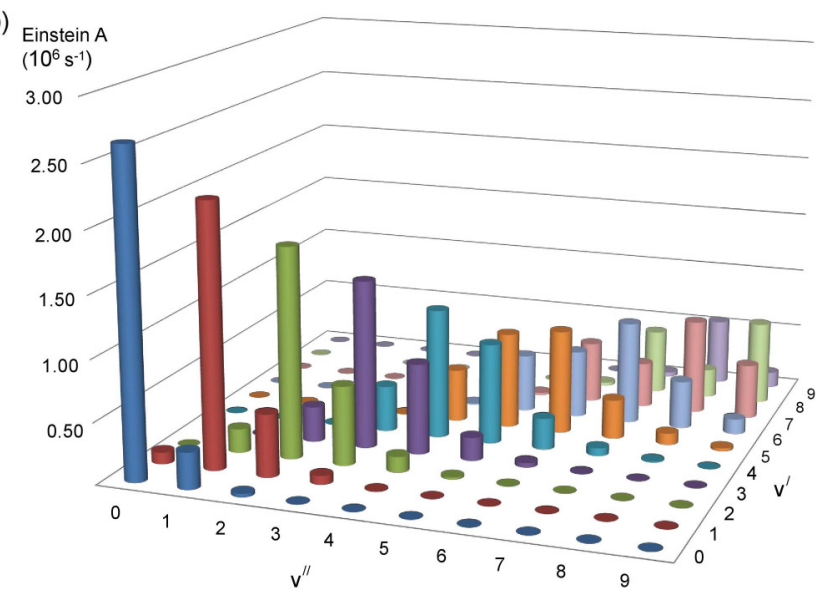

FIG. 7. (Color online) Comparison between (a) FC factors and (b) vibrational branching ratios for the $B{ }^{2} \Sigma^{+}-X^{2} \Sigma^{+}$transition in BaH.

the minimization of any losses (radiative and nonradiative). Computing the relevant FC factors are not enough when there exist more than one lower electronic state as is the case in $\mathrm{BaH}$. Consequently, we determined the ratio of the loss channels (a) nondiagonal $A^{2} \Pi_{1 / 2} v=0 \leftrightarrow X^{2} \Sigma^{+} v=1$, 2, or 3 ; (b) $B^{2} \Sigma^{+} v=0 \leftrightarrow X^{2} \Sigma^{+} v=1,2$, or 3 ; (c) $A^{2} \Pi_{1 / 2} v=$ $0 \leftrightarrow H^{2} \Delta_{3 / 2} \quad v=0$; and (d) $B^{2} \Sigma^{+} v=1 \leftrightarrow A^{2} \Pi_{1 / 2} v=0$, 1 , and 2 . Furthermore, the large vibrational energy separation in hydrides means that even within a single electronic manifold the FC factors fail to capture the large changes in vibrational branching ratios $R_{v^{\prime} v^{\prime \prime}}$, determined by the relative Einstein $A$ coefficients

$$
R_{v^{\prime} v^{\prime \prime}}=\frac{A_{v^{\prime} v^{\prime \prime}}}{\left(\sum_{v^{\prime \prime}} A_{v^{\prime} v^{\prime \prime}}\right)}
$$

because they do not account for the $\omega^{3}$ dependence of radiative decay. Radiative decay to higher vibrational levels with their corresponding lower emission frequencies are suppressed compared to the desired cyclic 0-0 transition
(Fig 7). This effect is particularly strong in hydrides because of the relatively large vibrational frequencies.

It was found that the $A^{2} \Pi-X^{2} \Sigma^{+}$transition has a number of advantages over its $B{ }^{2} \Sigma^{+}-X^{2} \Sigma^{+}$rival as the main cooling cycle, not least that the diagonal FC factors are slightly higher and the lifetime significantly shorter for a higher cooling rate. But it is the way the favorable nature of the $0-0$ transition is enhanced when the change in the transition frequency is taken into account that is particularly strong for the $A^{2} \Pi-X^{2} \Sigma^{+}$transition. For example, comparing the decay $A^{2} \Pi-X^{2} \Sigma^{+} 0-0$ and $0-1$ to the total loss from $v^{\prime}=0$ suggests that the cooling cycle will switch off in 3000 cycles when the FC factors alone are considered, but this rises more than tenfold to 40-60000 cycles if the change in frequency is taken into account as well. The wavelength effect is illustrated in Fig. 7 where the $B^{2} \Sigma^{+}-X^{2} \Sigma^{+}$FC factors are compared with branching ratios $R_{v^{\prime} v^{\prime \prime}}$; note the change in relative heights for the off-diagonal transitions $\Delta v= \pm 1$.

More importantly, the wavelength effect ensures the ratio between the primary $0-0 A^{2} \Pi-X^{2} \Sigma^{+}$cyclic decay channel (a)

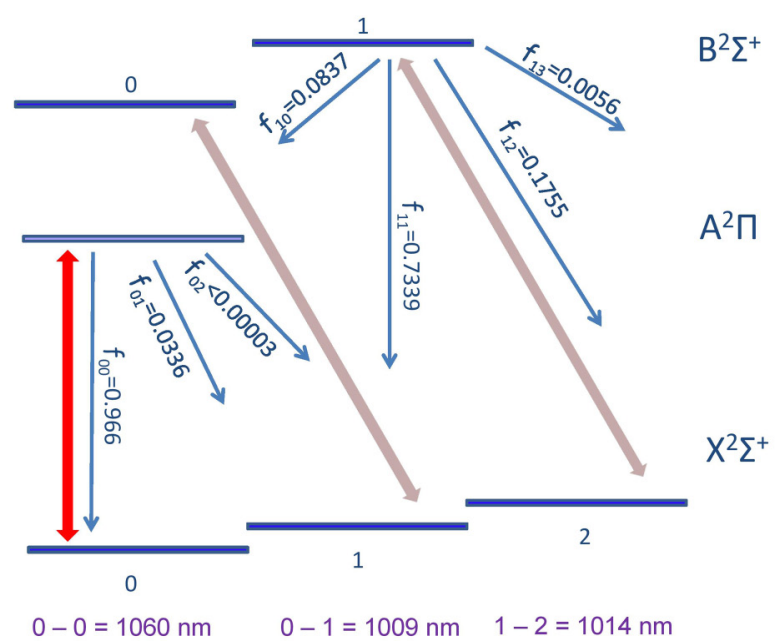

(b)

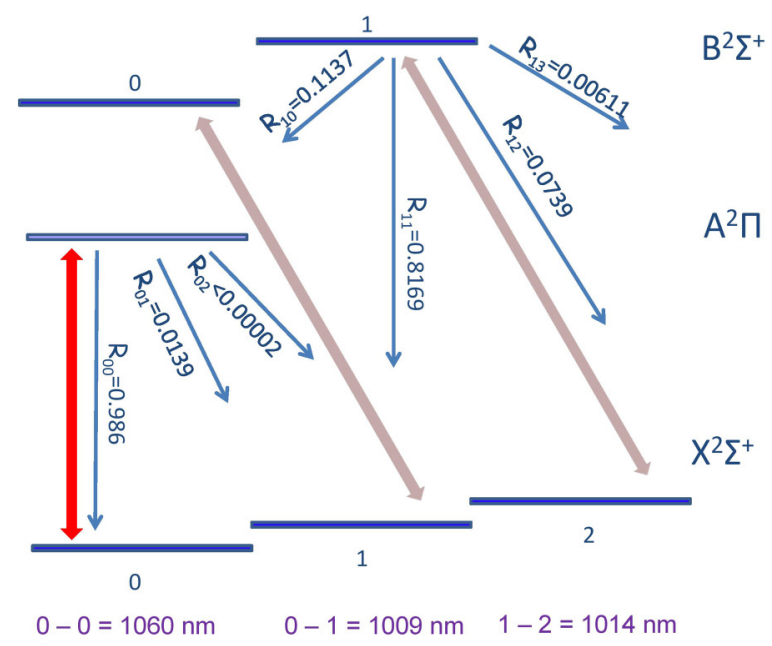

FIG. 8. (Color online) Comparison between (a) FC factors and (b) vibrational branching ratios for the proposed mixed $B^{2} \Sigma^{+}-X^{2} \Sigma^{+}$ and $A^{2} \Pi-X^{2} \Sigma^{+}$cooling cycle in BaH. In addition, the $1 \leftarrow 2$ transition can be removed and a closed cycle of greater than 30000 cooling transitions achievable in $\mathrm{BaH}$, though for the heavier $\mathrm{BaD}$ this reduces by more than a factor of 3 . 
and loss to the $v=0$ level of $H^{2} \Delta_{3 / 2}$ (the only accessible excited rovibronic level below $\left.A^{2} \Pi_{1 / 2} v=0\right)$ is $>150000: 1$ despite a fairly strong transition dipole for the latter transition (Fig. 4), therefore ensuring that this decay channel leads to negligible losses during cooling.

Another consideration is the availability of suitable cooling lasers. $1060 \mathrm{~nm}$ should be accessible by a fiber laser, which have considerable power and narrow linewidths. Instead of pumping on the $0-1 A^{2} \Pi-X^{2} \Sigma^{+}$transition to maintain the cooling cycle, which would lie further into the infrared, it is possible instead to pump on the $0-1 B^{2} \Sigma^{+}-X^{2} \Sigma^{+}$transition as this wavelength can be generated with a Ti:sapphire ring laser. Further experimental details such as beam sources, vibrational distributions, etc. can be found in the Supplemental Material [33].

The $a b$ initio results reveal that when cooling is primarily conducted on the $A^{2} \Pi_{1 / 2} v=0 \leftrightarrow X^{2} \Sigma^{+} v=0$ transition, only $A^{2} \Pi_{1 / 2} v=0 \leftrightarrow X^{2} \Sigma^{+} v=1$ contributes significantly to the loss. In conclusion, the cooling scheme in Fig. 8 is favored, though a reduced two color laser cooling scheme ( $>40000$ cooling cycles) is perfectly feasible. This wavelength suppression effect is a major advantage of cooling hydrides over other diatomic molecules. The smaller vibrational frequency in the deuteride weakens the effect of changes in emission wavelength, and the branching ratio for $A^{2} \Pi_{1 / 2} v=0 \leftrightarrow X^{2} \Sigma^{+} v=2$ is consequently five times higher in $\mathrm{BaD}$ than $\mathrm{BaH}$. Thus it is only possible to achieve 8-9000 cooling transitions with two laser colors. A recent ab initio calculation [35] using relativistic potentials (the discrepancies between the theoretical and experimental $r_{e}$ values $<0.01 \AA$ ) has computed the bond dissociation energy $D_{e}\left(X^{2} \Sigma^{+}\right)$as $16681 \mathrm{~cm}^{-1}(2.06 \mathrm{eV})$ which ensures that the lowest vibrational levels $v^{\prime}=0$ and 1 of the $B^{2} \Sigma^{+}$state are both below the ground dissociation limit. Therefore, predissociation is not a concern for laser cooling $\mathrm{BaH}$ with the scheme proposed.

Separate from the cooling efficiency, one final consideration is the need to ultimately trap the slow $\mathrm{BaH}$ molecules. The recent successful trapping [36] of $\mathrm{SrF}$ in a Magneto-Optical Trap (MOT) highlights the effect that the nature of the excited electronic state has on the trapping efficiency. In particular, the Zeeman splitting should exceed that of the ground state and due to a cancellation effect it seems likely that excited $A^{2} \Pi_{1 / 2}$ states, in general, are not ideal [37] for a MOT. Thanks to mixing with vibrational levels in the $B^{2} \Sigma^{+}$state because of the heavy $\mathrm{Ba}$ atom a reasonable trapping force will be available in $\mathrm{BaH}$ though the large rotational constant is a disadvantage for hydrides when very large couplings are desired. It may therefore prove more effective to trap BaH using the $B^{2} \Sigma^{+}-$ $X^{2} \Sigma^{+}$wavelength scheme in Fig. 6 instead.

\section{Zero-energy fragmentation pathway}

\section{Resonance linewidth}

To achieve zero-energy fragmentation of the parent, optical transfer into a Feshbach level just under the dissociation limit is promoted before fragmentation of the weakly bound state by tuning an external field. The energy width $\Gamma(E)$ of the
Feshbach resonance will ultimately determine the velocity distribution of the atomic fragments formed and depends on the coupling $W(R)$ between the closed and open scattering channels [38] (Fermi's golden rule)

$$
\Gamma(E)=2 \pi\left|\left\langle\Psi_{c}|W(R)| \Psi_{o}\right\rangle\right|^{2} .
$$

The interaction between an alkaline earth $\left({ }^{1} S_{0}\right)$ atom and a hydrogen atom results in a very weak coupling [39] between the molecular and atomic channels, creating the sharp Feshbach resonances required (a closed-channel dominated resonance) to ensure the linewidth $\Gamma(E)$ corresponds to sub$\mu K$ temperatures in accordance with Eq. (7). Specifically it is the change in the atomic hyperfine coupling interaction due to the presence of the second atom that is the largest contributor to the coupling. Thanks to the proton spin this coupling due to $\Delta \zeta_{H}(R)$ is always present in diatomic hydrides.

When considering experimental implementation of the technique, a magnetic field is usually used for the dissociation step so it is the final width of the resonance in terms of the magnetic field $\Delta(B)$ and not $\Gamma(E)$ that is the crucial experimental parameter. The magnetic field will be a component in the Hamiltonian describing the coupling $W(R)$ but as Eq. (7) demonstrates there is also a transformation of the observed linewidth from the energy to magnetic field scales. To ensure [40] the magnetic width is not too narrow, it should be advantageous to minimize the value of the product $\left(2 m_{\mu}\right)^{1 / 2} a_{\mathrm{bg}} \delta \mu$. As discussed earlier, it is inevitable that the value of $m_{\mu}$ is close to 1 for a heavy hydride. It is presently only possible to obtain a reliable value for $a_{\mathrm{bg}}$ from experimental measurements with ultracold atoms but it has been shown that there is a $75 \%$ chance of it lying between $-\bar{a}$ and $3 \bar{a}$, where $\bar{a}$ is the mean scattering length which is known [41] to be proportional to $\left(2 m_{\mu} C_{6}\right)^{1 / 4}$. Using the earlier dispersion constant, the estimated $a_{\mathrm{bg}}$ value for $\mathrm{BaH}(\mathrm{BaD})$ lies between $-13 a_{0}$ and $39 a_{0}\left(-15 a_{0}\right.$ and $\left.45 a_{0}\right)$, a comparable range to $\mathrm{LiYb}$ [39] and at the lower end of known scattering lengths. This low value again appears to be a general feature of hydrides (thanks to the small values of $m_{\mu}$ and $C_{6}$ ). Finally, a small value of $\delta \mu$ requires a small difference in the energy shift with magnetic field between the open and closed scattering channels. In the case of alkali molecules $\delta \mu \sim 2 \mu$ atom because the closed (molecular) scattering channel has no nonnuclear magnetic dipole as it is a singlet state $(S=0)$. In $\mathrm{BaH}$ the nonzero electron spin should ensure that it is possible to find resonances with a range of values for $\delta \mu$, including values $\delta \mu \ll 1$. In summary, a relatively large $\Delta(B)$ can correspond to a small energy release $\Gamma(E)$ in hydrides like $\mathrm{BaH}$.

\section{Isotope effects}

A further opportunity to ensure the ideal Feshbach resonance is found can be exploited by considering the different isotopes available as the coupling $W(R)$ is affected through changes in hyperfine constants. $\mathrm{BaH}$ has a number of odd and even isotopes but due to their larger abundances the focus here will be ${ }^{137} \mathrm{BaH}$ and ${ }^{138} \mathrm{BaH}$. Even-mass isotopes of the ${ }^{1} S_{0}$ atoms are usually without nuclear spin and cannot contribute to the Feshbach linewidth. This is not the case for odd isotopes that can also couple to the molecular spin [39] via 
$\Delta \zeta_{\mathrm{Ba}}(R)$. For the corresponding alkali-alkaline earth diatomics ${ }^{7} \mathrm{Li}^{173} \mathrm{Yb}$ and ${ }^{7} \mathrm{Li}^{174} \mathrm{Yb}$, the observed widths are typically $100 \times$ larger for the ${ }^{173} \mathrm{Yb}$ species but the resonance width is typically $<5 \mathrm{mG}$ even for the broadest lines. This suggests the analogous ${ }^{137} \mathrm{BaH}$ is a potentially attractive candidate for ultracold hydrogen production. Compared to ${ }^{6} \mathrm{Li}^{173} \mathrm{Yb}$ there will be fewer Feshbach resonances in ${ }^{137} \mathrm{BaH}$ because of the smaller nuclear spin in ${ }^{137} \mathrm{Ba}(I=1 / 2$ versus $I=5 / 2$ in ${ }^{173} \mathrm{Yb}$ ) and the Feshbach widths will be relatively insensitive to the value of $m_{i, \mathrm{Ba}}$ because of the corresponding small changes in the crucial $\frac{1}{2}\left[i_{\mathrm{Ba}}\left(i_{\mathrm{Ba}}+1\right)-m_{i, \mathrm{Ba}}\left(m_{i, \mathrm{Ba}}+1\right)\right]^{1 / 2}$ coupling [39] term. However, it is important to appreciate that the value of $\Delta \zeta_{H}(R)$ is larger in both absolute and relative terms than the equivalent change for ${ }^{7} \mathrm{Li}$ or ${ }^{137} \mathrm{Yb}$ atoms in $\mathrm{LiYb}$, falling from the atomic value of 1420 to [42] $47 \mathrm{MHz}$ in ${ }^{138} \mathrm{BaH}$ at the equilibrium bond length, just $3 \%$ of an isolated $\mathrm{H}$ atom.

Turning to the molecular hyperfine structure in the lowest $N=0$ state, ${ }^{138} \mathrm{BaH}$ has two hyperfine levels $F=0$ or 1 while ${ }^{138} \mathrm{BaH} F=1 / 2$ or $3 / 2$. Assuming that only molecules in the lowest hyperfine state are trapped, the narrowest resonance in ${ }^{138} \mathrm{BaD}$ it will be $m_{f D}=+\frac{1}{2}$ (there will be an additional resonance for $m_{f D}=-\frac{1}{2}$ ) while in ${ }^{138} \mathrm{BaH}$ there is only $m_{f H}=0 . \Delta(B)$ scales as $B^{2}$ for low magnetic fields before leveling out, but this effect will be counteracted by the shallower energy slopes in hydrogen at lower fields [43] for $m_{f H}=0\left(m_{f D}=+\frac{1}{2}\right)$ below $500 \mathrm{G}(100 \mathrm{G})$. According to Eq. (7), in order to get the full kinematic effect, with a final hydrogen "temperature" of $\sim 200 \mathrm{nK}$ from Doppler cooled BaH, a resonance width of around $\Delta(B)=2-100 \mathrm{mG}$ is required, depending on the value of $\delta \mu$. The lower end of this range is challenging still for experiments but the magnetic field control demonstrated in recent work on ultracold dysprosium [44] is certainly compatible with the strict demands here, where Feshbach resonances of $\sim 10 \mathrm{mG}$ width were observed with at a resolution of just $1.8 \mathrm{mG}$.

\section{STIRAP pathway}

To ensure that optical transfer is possible, a pathway to the highest bound levels of the $X^{2} \Sigma^{+}$state is found using the $a b$ initio potentials. Despite lying thousands of wave numbers above $v=0$, the highest (final) vibrational level close to the dissociation limit will have a significantly longer radiative lifetime [45] $(\approx 0.1-1000$ s depending on the binding energy) than all the other excited vibrational levels, even the first excited $v=1$ state, while the inelastic collision rate constant [46] is typically only of the order $10^{-11} \mathrm{~cm}^{3} \mathrm{~s}^{-1}$. As background decay pathways can negatively impact the efficiency of STIRAP processes it would seem advisable to avoid as much of the Rydberg structure as possible. Bearing in mind that the ionization energy of Ba has been recorded [47] as $42035 \mathrm{~cm}^{-1}$ and the bond strength calculated [35] as $16631 \mathrm{~cm}^{-1}$, the upper STIRAP state was limited to those involving the ${ }^{3} P_{0}$ and ${ }^{3} P_{1}$ states of the product $\mathrm{Ba}$ atom. The ${ }^{3} P_{0}$ and ${ }^{3} P_{1}$ asymptotes lie, respectively, 28897 and $29268 \mathrm{~cm}^{-1}$ above the ground state minimum. The latest calculated bond strengths $[48,49]$ of $\mathrm{BaH}^{+}$differ by nearly $2000 \mathrm{~cm}^{-1}$ (21 604 versus $19596 \mathrm{~cm}^{-1}$ ) so the best estimate of the adiabatic ionization energy of $\mathrm{BaH}$ is $37062-39070 \mathrm{~cm}^{-1}$, well above these asymptotes. One

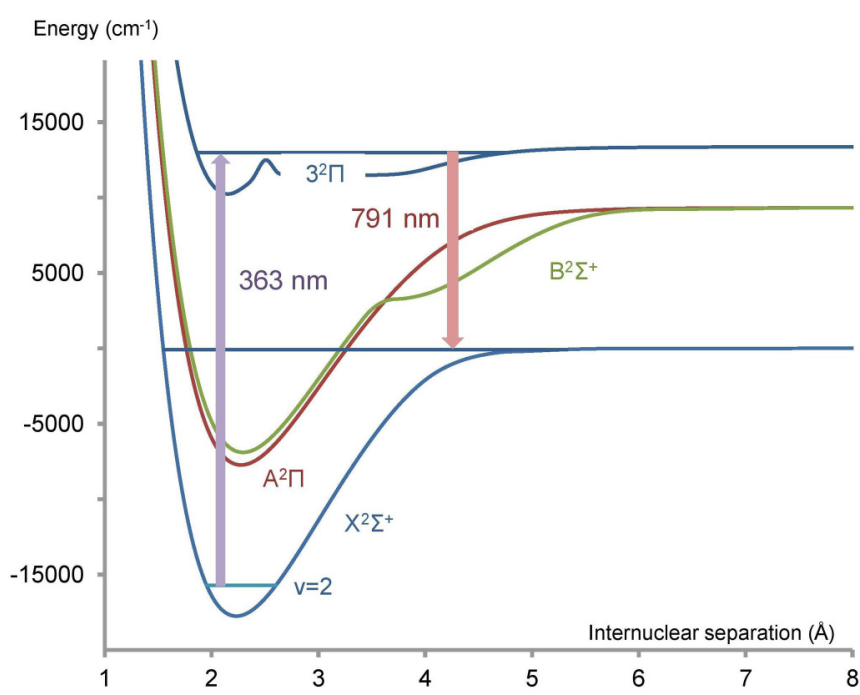

FIG. 9. (Color online) Proposed STIRAP pathway in BaH and the corresponding pulse wavelengths. Also marked are the bound state potentials involved in the cooling cycle.

possible STIRAP pathway is illustrated in Fig. 9 and involves the $3^{2} \Pi-X^{2} \Sigma^{+}$transition from $v=2$ in the $X^{2} \Sigma^{+}$state. The Einstein $A$ coefficients for both the PUMP and DUMP transitions are healthy $\left(>10^{4} \mathrm{~s}^{-1}\right)$ and the required laser pulses can be produced by a variety of laser sources including high-powered diode lasers or an Alexandrite solid state laser.

Recently, Zare and co-workers have proposed a simpler alternative to STIRAP that can be performed with UV and visible laser pulses with peak intensities $\leqslant 20 \mathrm{GW} \mathrm{cm}^{-2}$. This Stark-induced adiabatic Raman passage (SARP) has achieved 97\% population transfer between $v=0$ and $v=1$ [50] of $\mathrm{H}_{2}$ in a molecular beam but simulations suggest that vibrational changes of at least $\Delta v=4$ are perfectly feasible. Two advantages of SARP are that you do not need an intermediate vibronic level and the transfer process is complete within around $20 \mathrm{~ns}$.

The final dissociation of the Feshbach can be initiated by sweeping the resonance across the dissociation threshold by means of an external field. The required scanning rate to ensure close to $100 \%$ transfer from the molecular state to the lowest kinetic energy state of the atoms can be estimated using a simple model where the continuum of final kinetic energies is replaced by quantized energy states. These are not the real trapping states, which are set by the external fields defining the trap, but representations of the center of mass energy release required by Eq. (7),

$$
\frac{T_{D}}{138} \approx 190 \mathrm{nK} \approx 4.4 \mathrm{kHz},
$$

setting the energy separation $h v$. The upper bound of the scanning rate is determined by the desire to minimize the populations of excited kinetic states $2 h v, 3 h v$, etc. during the fragmentation step. This ensures that the molecular Feshbach is adiabatically transformed into the slowest atoms and can be estimated by a simple Landau-Zener (LZ) model [51], specifically by ensuring that the Landau-Zener parameter 
(a)

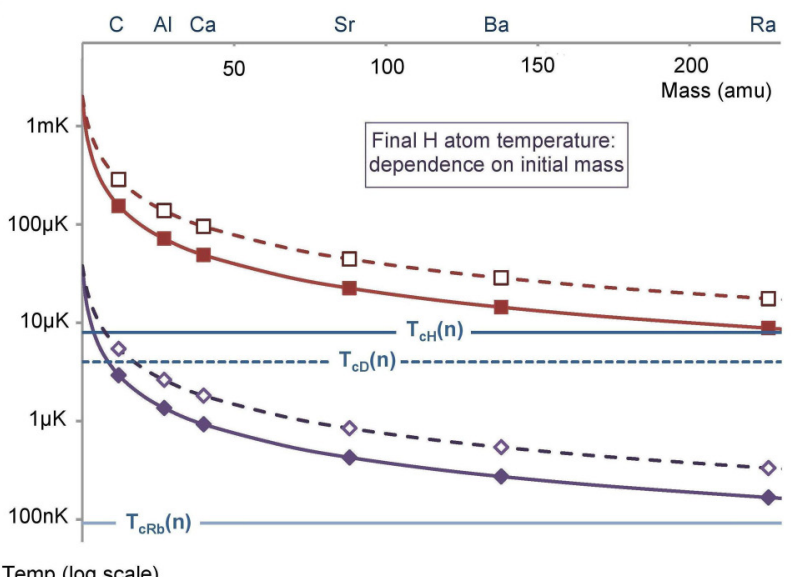

(b)

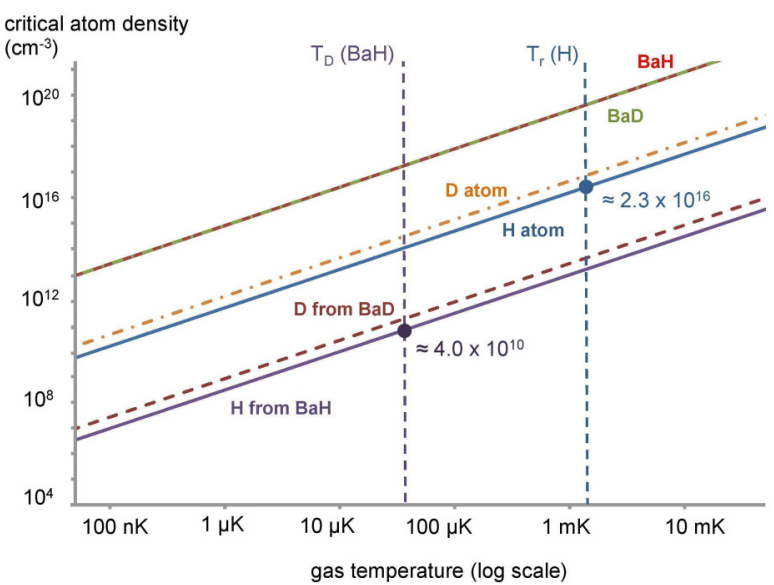

FIG. 10. (Color online) The effect of parent mass on the final $\mathrm{H}$ atom temperatures following molecular fragmentation. (a) For molecules with initial temperature $26 \mu \mathrm{K}$ or $2 \mathrm{mK}$ prior to fragmentation (the center of mass fragmentation energy must be considerably smaller than the thermal energy of the parents). Dashed lines correspond to the deuterated species. Also marked are the values of $T_{c}$ for $\mathrm{Rb}, \mathrm{D}$, and $\mathrm{H}$ at the experimental density [52] $n$ used by Hadzibabic and co-workers. (b) The effect of mass on the critical density to achieve a quantum gas, compared with direct cooling of $\mathrm{H}$ or $\mathrm{D}$ atoms alone. Also marked are the recoil temperature for $\mathrm{H}$ atom cooling and the Doppler temperature for $\mathrm{BaH} A^{2} \Pi_{1 / 2} \leftrightarrow X^{2} \Sigma^{+}$cooling and the corresponding critical densities (in $\mathrm{cm}^{-3}$ ). The origin of the significant reduction in critical density is discussed in the text.

$W_{0, \text { Fesh }}^{\mathrm{LZ}}$ greatly exceeds unity

$$
\left(T_{\text {res }}\right)(2 \pi v)=W_{0, \text { Fesh }}^{\mathrm{LZ}} \gg 1 .
$$

$T_{\text {res }}$ is the minimum duration through the resonance to ensure adiabaticity. Using $v \approx 4.4 \mathrm{kHz}, T_{\text {res }} \gg 3.6 \mu \mathrm{s}$, which is therefore also the minimum lifetime of the Feshbach required and is clearly much shorter than the expected radiative lifetime. Even for the range (2-200 $\mathrm{mG}$ ) of Feshbach resonances considered here, the required scanning rate of a dissociating magnetic field $\Gamma_{\text {scan }} \ll 10^{2}-10^{4} \mathrm{G} \mathrm{s}^{-1}$ is a condition that is not difficult to meet.

\section{CONDENSATION BY FRAGMENTATION}

Figure 10(a) presents the temperature dependence of the final hydrogen (and deuterium) fragments on the mass of the parent molecules assuming the velocity distribution of the hydrogen fragments matches that of the parents. Recently a nearly uniform atom trap has been developed by Hadzibabic and co-workers for the production of a Rb BEC and an accurate transition temperature of $T_{c \mathrm{Rb}}=92 \pm 3 \mathrm{nK}$ measured [52] for $n \approx 3 \times 10^{12}$ atoms cm$~^{-3}$. The general dependence of $T_{\text {c.m. }}$ on particle mass $m$ and density $n$ is $T_{\text {c.m. }} \propto n^{2 / 3} / m$ so using the experimental result for $\mathrm{Rb}$ and assuming an identical particle density, the corresponding transition temperatures for $\mathrm{H}$ and $\mathrm{D}$ atoms, $T_{c H}$ and $T_{c D}$, are added to Fig. 10(a). The $\mathrm{H}$ (D) atoms from zero-energy fragmentation of $\mathrm{CaH}(\mathrm{CaD})$ and heavier hydrides are at a lower temperature than $T_{c H(D)}$ when the parent temperature is approximately in the range $20-100 \mu \mathrm{K}$.

The above particle density is rather optimistic compared to current expectations for ultracold molecular traps but perhaps the most significant benefit of the fragmentation technique is the large reduction in the critical density required for quantum degeneracy at a fixed temperature $T$. The mass dependence of critical density is $(m T)^{3 / 2}=n$, where $n$ is the density of the gas in particles $\mathrm{cm}^{-3}$ and $m$ is the mass, so for two species $\left(m_{2}>\right.$ $\left.m_{1}\right)$ at a fixed temperature, the ratio of critical densities is

$$
\frac{n_{2}}{n_{1}}=\left(\frac{m_{2}}{m_{1}}\right)^{3 / 2} \text {. }
$$

A heavier species will naturally have a larger critical density at a given temperature. Zero-energy fragmentation of the hydride, however, leads to a larger de Broglie wavelength for the hydrogen atoms than the parent molecules $\lambda_{H} \approx$ $m_{M H} \lambda_{M H}$, increasing the phase space volume. Thus the ratio of critical densities for a molecule $m_{M H}$ that fragments with zero-energy release into atoms to the critical density of the hydrogen fragments is simply the cube of the de Broglie boost

$$
\frac{n_{H^{\prime}}}{n_{M H}}=\left(\frac{m_{H}}{m_{M H}}\right)^{3} .
$$

Consequently, the ratio of critical densities $n_{H}$ for an atomic hydrogen gas and hydrogen fragmentation products $n_{H}^{\prime}$ at a fixed $T$ is

$$
\frac{n_{H^{\prime}}}{n_{H}}=\left(\frac{m_{H}}{m_{M H}}\right)^{3}\left(\frac{m_{M H}}{m_{H}}\right)^{3 / 2}=\left(\frac{m_{H}}{m_{M H}}\right)^{3 / 2} .
$$

Figure 10(b) documents the critical density as a function of the initial temperature for a gas of hydrogen atoms, $\mathrm{BaH}$ molecules, and for the hydrogen atoms produced by fragmentation of the same $\mathrm{BaH}$ gas. Although the above technique is most effective for production of cold $\mathrm{H}$ or $\mathrm{D}$, in principle it can offer advantages for any atom forming a diatomic with a heavier partner, particularly for the lighter elements along the second row. Consequently, if narrow Feshbach resonances can be found for a ${ }^{2} P$ or ${ }^{3} P$ atom with 
a ${ }^{1} S$ alkaline earth, then the production of ultracold carbon, oxygen (both ${ }^{3} P$ ) and fluorine $\left({ }^{2} P\right)$ is helped by pairing with a heavy alkaline earth. So while with BeF there will be no cooling effect, $\mathrm{SrF}$ will produce a $107 / 19 \approx 5.6$ times drop in final fluorine gas temperature.

The recoil $T_{r}(H)$ and Doppler $T_{D}(H)$ temperatures of laser cooled hydrogen atoms are both rather high, above $1 \mathrm{mK}$, ensuring a relatively large critical density compared to laser cooled alkalis. Another advantage of the fragmentation scheme is the possibility of creating a much lower temperature sample by exploiting the visible cooling transitions and larger masses of molecular species. By exploiting the twin effects of the $\left(m_{H} / m_{M H}\right)^{3 / 2}$ mass dependence (at a fixed temperature) in combination with the lower temperatures possible by laser cooling the parent molecule compared to $\mathrm{H}$ atoms directly $\left[T_{r}(\mathrm{BaH}) \ll T_{D}(\mathrm{BaH}) \ll T_{r}(H) \approx T_{D}(H)\right]$, a dramatic fall in the hydrogen critical density may be achieved. This demonstrates that a relatively dilute gas of ultracold $\mathrm{H}$ below $T_{c H}$ can be created with benefits such as a longer lifetime and smaller collisional shifts. Although a $26 \mu \mathrm{K}$ gas temperature $\left[T_{D}(\mathrm{BaH})\right.$ for the $\mathrm{A}^{2} \Pi \leftrightarrow X^{2} \Sigma^{+}$transition] is not demanding for an atomic gas, to date the lowest temperature achieved by direct laser cooling of a molecular sample [53] (YO) is around $2 \mathrm{mK}$. However, this is comparable with $T_{r}(H)$ by direct laser cooling of atomic hydrogen and by photodissociating a gas of $\mathrm{BaH}$ at this temperature the critical density is some $(139)^{3 / 2} \sim 1600$ times smaller.

\section{SUMMARY AND CONCLUSION}

An ultracold gas of hydrogen atoms would be the ultimate laboratory to probe fundamental questions about our universe. In this paper a technique to create ultracold hydrogen and, uniquely, deuterium atoms by the fragmentation of ultracold and trapped diatomics has been analyzed. Four important conditions that the molecular parent should fulfill in order to achieve the lowest possible temperatures have been identified. Using $a b$ initio molecular potentials and the transition dipoles of $\mathrm{BaH}$ relevant for the production of ultracold $\mathrm{H}$ via fragmentation of laser cooled molecules, the most efficient laser cooling scheme and principal decay channels have been found. Naturally, a proof of principle experiment could be performed on a trapped cold gas of $\mathrm{BaH}$ well above $T_{D}$, for then the kinematic effect could be observed using much broader Feshbach resonances than discussed in the text.

A single-color scheme for $\mathrm{BaH}$ will ensure 50-70 optical cycles (which should be observable in experimental setups like Hummon et al. [53]) a two-color scheme 40-50000, while a three-color experiment is effective for $>10^{6}$ cycles. A STIRAP excitation process via the $3{ }^{2} \Pi$ state (a mixed Rydberg/valence state) appears feasible with good oscillator strengths. Exploiting the $m_{H(D)} / m_{M H(D)}$ dependence of the final temperature when $v_{\text {c.m. }}$. $\ll v_{M H(D)}$ it is possible to create a dilute gas of hydrogen or deuterium below $T_{c}$ with full optical access and a reduction of the critical density by a factor of at least $10^{5}$ compared to directly laser cooled hydrogen atoms.
[1] T. Hänsch and A. Schawlow, Opt. Commun. 13, 68 (1975).

[2] I. D. Setija, H. G. C. Werij, O. J. Luiten, M. W. Reynolds, T. W. Hijmans, and J. T. M. Walraven, Phys. Rev. Lett. 70, 2257 (1993).

[3] D. G. Fried, T. C. Killian, L. Willmann, D. Landhuis, S. C. Moss, D. Kleppner, and T. J. Greytak, Phys. Rev. Lett. 81, 3811 (1998).

[4] T. C. Killian, D. G. Fried, L. Willmann, D. Landhuis, S. C. Moss, T. J. Greytak, and D. Kleppner, Phys. Rev. Lett. 81, 3807 (1998).

[5] N. P. Wells and I. C. Lane, Phys. Chem. Chem. Phys. 13, 19036 (2011).

[6] I. C. Lane, Phys. Chem. Chem. Phys. 14, 15078 (2012).

[7] K. Muller-Dethlefs and E. W. Schlag, Annu. Rev. Phys. Chem. 42, 109 (1991).

[8] M. Grechko, O. V. Boyarkin, T. R. Rizzo, P. Maksyutenko, N. F. Zobov, S. V. Shirin, L. Lodi, J. Tennyson, A. G. Császár, and O. L. Polyansky, J. Chem. Phys. 131, 221105 (2009).

[9] E. S. Shuman, J. F. Barry, and D. DeMille, Nature (London) 467, 820 (2010).

[10] S. D. Hoga and F. Merkt, Phys. Rev. Lett. 100, 043001 (2008).

[11] S. D. Hogan, A. W. Wiederkehr, H. Schmutz, and F. Merkt, Phys. Rev. Lett. 101, 143001 (2008).

[12] A. J. Moerdijk, B. J. Verhaar, and A. Axelsson, Phys. Rev. A 51, 4852 (1995).

[13] J. D. Danzl et al., Nat. Phys. 6, 265 (2010).

[14] S. Inouye, M. R. Andrews, J. Stenger, H.-J. Miesner, D. M. Stamper-Kurn, and W. Ketterle, Nature (London) 392, 151 (1998).
[15] P. S. Zuchowski, J. Aldegunde, and J. M. Hutson, Phys. Rev. Lett. 105, 153201 (2010).

[16] C. Chin, R. Grimm, P. Julienne, and E. Tiesinga, Rev. Mod. Phys. 82, 1225 (2010).

[17] I. Prigogine and E. Xhrouet, Physica 15, 913 (1949).

[18] N, V. Vitanov, T. Halfmann, B. W. Shore, and K. Bergmann, Annu. Rev. Phys. Chem. 52, 763 (2001).

[19] Y. Gao and T. Gao, Phys. Rev. A. 90, 052506 (2014).

[20] B. Yadin et al., Mon. Not. R. Astron. Soc. 425, 34 (2012).

[21] N. P. Wells and I. C. Lane, Phys. Chem. Chem. Phys. 13, 19018 (2011).

[22] H. -J. Werner, P. J. Knowles, R. Lindh, F. R. Manby, M. Schútz, P. Celani, T. Korona, A. Mitrushenkov, G. Rauhut, T. B. Adler, R. D. Amos, A. Bernhardsson, A. Berning, D. L. Cooper, M. J. O. Deegan, A. J. Dobbyn, F. Eckert, E. Goll, C. Hampel, G. Hetzer, T. Hrenar, G. Knizia, C. Köppl, Y. Liu, A. W. Lloyd, R. A. Mata, A. J. May, S. J. McNicholas, W. Meyer, M. E. Mura, A. Nicklass, P. Palmieri, K. Pflüger, R. Pitzer, M. Reiher, U. Schumann, H. Stoll, A. J. Stone, R. Tarroni, T. Thorsteinsson, M. Wang, and A. Wolf, MOLPRO, version 2010.1, a package of $a b$ initio programs (2009).

[23] H.-J. Werner and P. J. Knowles, J. Chem. Phys. 82, 5053 (1985).

[24] H.-J. Werner and P. J. Knowles, J. Chem. Phys. 89, 5803 (1988).

[25] S. R. Langhoff and E. R. Davidson, Int. J. Quantum Chem. 8, 61 (1974).

[26] R. J. Le Roy, LEVEL 8.0: A computer program for solving the radial Schrödinger equation for bound and quasibound levels, Chemical Physics Research Report CP-663 (University of Waterloo, 2007) the source code and manual for this program may be obtained fromhttp://leroy.uwaterloo.ca/programs/. 
[27] M. V. Ramanaiah and S. V. J. Lakshman, Physica B+C 113, 263 (1982).

[28] R. S. Ram and P. F. Bernath, J. Mol. Spectrosc. 283, 18 (2013).

[29] A. Bernard, C. Effantin, J. D'incan, G. Fabre, R. Stringat, and R. F. Barrow, Mol. Phys. 67, 1 (1989).

[30] O. Appelblad, L.-E. Berg, L. Klynning, and J. W. C. Johns, Phys. Scr. 31, 69 (1985).

[31] G. Fabret, A. El-Hachimi, R. Stringat, C. Effantin, A. Bernard, J. D'Incan, and J. Verges, J. Phys. B: At. Mol. Phys. 20, 1933 (1987).

[32] A. Derevianko, S. G. Porsev, and J. F. Babb, At. Data Nucl. Data Tables 96, 323 (2010).

[33] See Supplemental Material at http://link.aps.org/supplemental/ 10.1103/PhysRevA.92.022511 for further information on BaH dispersion coefficients, temperature effects on vibrational and rotational populations, details of the two-color cooling schemes, and tables of FC factors for the cooling transitions.

[34] L.-E. Berg, K. Ekvall, A. Hishikawa, and S. Kelly, Phys. Scr. 55, 269 (1997).

[35] L. V. Skripnikov, N. S. Mosyagin, and A. V. Titov, Chem. Phys. Lett. 555, 79 (2013).

[36] D. J. McCarron, E. B. Norrgard, M. H. Steinecker, and D. DeMille, New J. Phys. 17, 035014 (2015).

[37] M. R. Tarbutt, New J. Phys. 17, 015007 (2015).

[38] H. Beutler, Z. Phys. 93, 177 (1935); U. Fano, Nuovo Cimento 12, 154 (1935); Phys. Rev. 124, 1866 (1961).

[39] D. A. Brue and J.M. Hutson, Phys. Rev. Lett. 108, 043201 (2012).
[40] D. A. Brue and J.M. Hutson, Phys. Rev. A 87, 052709 (2013).

[41] G. F Gribakin and V.V. Flambaum, Phys. Rev. A 48, 546 (1993).

[42] L. B. Knight, Jr., and W. Weltner, Jr., J. Chem. Phys. 54, 3875 (1971).

[43] D. Cheever et al., Nucl. Instrum. Methods A 556, 410 (2006).

[44] K. Baumann, N. Q. Burdick, M. Lu, and B. L. Lev, Phys. Rev. A 89, 020701(R) (2014).

[45] W. C. Stwalley and W. T. Zemke, Int. J. Quantum Chem. 10, 223 (1976).

[46] J. Deiglmayr, M. Repp, O. Dulieu, R. Wester, and M. Weidemüller, Eur. Phys. J. D 65, 99 (2011).

[47] C. E. Moore, Atomic Energy Levels, Vols. 1-3, Circ. No. 467 (Natl. Bur. Stand., Washington, DC, 1958), available athttp://www.nist.gov/srd/nsrds.cfm

[48] M. Aymer and O. Dulieu, J. Phys. B: At. Mol. Opt. Phys. 45, 215103 (2012).

[49] L. Mejrissi, H. Habli, H. Ghalla, B. Oujia, and F. X. Gadéa, J. Phys. Chem. A, 117, 5503 (2013).

[50] W. Dong, N. Mukherjee, and R. N. Zare, J. Chem. Phys. 139, 074204 (2013).

[51] F. H. Mies, E. Tiesinga, and P. S. Julienne, Phys. Rev. A 61, 022721 (2000).

[52] A. L. Gaunt, T. F. Schmidutz, I. Gotlibovych, R. P. Smith, and Z. Hadzibabic, Phys. Rev. Lett. 110, 200406 (2013).

[53] M. T. Hummon, M. Yeo, B. K. Stuhl, A. L. Collopy, Y. Xia, and J. Ye, Phys. Rev. Lett. 110, 143001 (2013). 